Cemil TunÇ

\title{
INSTABILITY IN MULTI DELAY FUNCTIONAL DIFFERENTIAL EQUATIONS OF FOURTH ORDER
}

\begin{abstract}
A vector functional differential equation of the fourth order with multiple deviating arguments is considered. New sufficient conditions are established to guarantee the instability of the zero solution of the equation to be considered. We give an example to illustrate the subject.

KEY WORDS: vector functional differential equation, fourth order, instability, multi delay.
\end{abstract}

AMS Mathematics Subject Classification: $34 \mathrm{~K} 20$.

\section{Introduction}

Time delay as a source of instability often appears in many dynamic systems, for example, biological systems, chemical systems, metallurgical processing systems, nuclear reactor, electrical networks etc.. Many researchers have paid a lot of attentions on the problem of instability of solutions of various scalar and vector differential equations of the fourth order with and without delay, in the literature. In the ordinary case, for some recent results to the instability of the fourth order differential equations the reader can refer to Ezeilo [2, 3, 4], Sadek [6], Sun and Hou [7], Tunç [8-14, 16] and the references thereof.

However, to the best of our knowledge from the literature, the instability of solutions for the vector differential equations of the fourth order with multiple deviating arguments has not been discussed in the literature. In this paper, our purpose is to establish a result for the instability in multi delay functional differential equations of the fourth order.

In ordinary case, some results with respect to the instability of the solutions can be summarized as the following:

First, Sun and Hou [7] took into consideration the scalar differential equation of the fourth order:

$$
x^{(4)}+a_{1} \dddot{x}+h(\dot{x}) \ddot{x}+g(x) \dot{x}+f(x)=0,
$$


where $a_{1}$ is a constant. The authors established some sufficient condition, which guarantee the instability of the solution $x=0$ of the above equation.

Later, Tunç $[14,16]$ considered the fourth order vector differential equations of the form

$$
X^{(4)}+A \dddot{X}+H(\dot{X}) \ddot{X}+G(X) \dot{X}+F(X)=0
$$

and

$$
X^{(4)}+A \dddot{X}+H(\dot{X}) \ddot{X}+G(X) \dot{X}+F(X(t-\tau))=0
$$

with and without delay, respectively. The author established sufficient condition to guarantee the instability of zero solution of the above differential equations.

In this paper, we consider the vector differential equation of the fourth order with multiple constant deviating arguments

$$
X^{(4)}+A \dddot{X}+H(\dot{X}) \ddot{X}+G(X) \dot{X}+F(X)+\sum_{i=1}^{n} E_{i}\left(X\left(t-\tau_{i}\right)\right)=0,
$$

where $X \in \Re^{n}, \tau_{i}$ are certain positive constants, the fixed delays, $t-\tau_{i}>0$, $A$ is an $n \times n$ - symmetric matrix, $H$ and $G$ are continuous $n \times n-$ symmetric matrices for the arguments displayed explicitly, $F: \Re^{n} \rightarrow \Re^{n}, E_{i}: \Re^{n} \rightarrow \Re^{n}$, $F(0)=E_{i}(0)=0, F$ and $E_{i}$ are continuous for all their arguments. The existence and uniqueness of the solutions of Eq. (1) is assumed.

Equation (1) is the vector version for systems of real fourth order nonlinear differential equations of the form:

$$
\begin{aligned}
x_{i}^{(4)} & +\sum_{k=1}^{n} a_{i k} x_{k}^{\prime \prime \prime}+\sum_{k=1}^{n} h_{i k}\left(x_{1}^{\prime}, \ldots, x_{n}^{\prime}\right) x_{k}^{\prime \prime} \\
& +\sum_{k=1}^{n} g_{i k}\left(x_{1}, \ldots, x_{n}\right) x_{k}^{\prime}+f_{i}\left(x_{1}, x_{2}, \ldots, x_{n}\right) \\
& +\sum_{i=1}^{n} e_{i}\left(x_{1}\left(t-\tau_{i}\right), \ldots, x_{n}\left(t-\tau_{i}\right)\right)=0, \quad i=1,2, \ldots, n .
\end{aligned}
$$

Instead of Eq. (1), we consider its differential system form

$$
\begin{aligned}
\dot{X}= & Y, \dot{Y}=Z, \dot{Z}=U, \\
\dot{U}= & -A U-H(Y) Z-G(X) Y-F(X) \\
& -\sum_{i=1}^{n} E_{i}(X)+\sum_{i=1}^{n} \int_{t-\tau_{i}}^{t} J_{E_{i}}(X(s)) Y(s) d s,
\end{aligned}
$$

which was obtained by setting $\dot{X}=Y, \ddot{X}=Z, \dddot{X}=U$ from Eq. (1). 
Let $J_{E_{i}}(X), J_{F}(X)$ and $J(H(Y) Y \mid Y)$ denote the linear operators from $E_{i}(X), F(X)$ and $H(Y)$ to

$$
\begin{gathered}
J_{E_{1}}(X)=\left(\frac{\partial e_{1 i}}{\partial x_{j}}\right), \ldots, J_{E_{n}}(X)=\left(\frac{\partial e_{n i}}{\partial x_{j}}\right), \\
J_{F}(X)=\left(\frac{\partial f_{i}}{\partial x_{j}}\right)
\end{gathered}
$$

and

$$
J(H(Y) Y \mid Y)=\left(\frac{\partial}{\partial y_{j}} \sum_{k=1}^{n} h_{i k} y_{k}\right)=H(Y)+\left(\sum_{k=1}^{n} \frac{\partial h_{i k}}{\partial y_{j}} y_{k}\right),
$$

$i, j=1,2, \ldots, n$, where $\left(x_{1}, \ldots, x_{n}\right),\left(y_{1}, \ldots, y_{n}\right),\left(e_{1 i}\right), \ldots,\left(e_{n i}\right),\left(f_{1}, \ldots, f_{n}\right)$ and $\left(h_{i k}\right)$ are components of $X, Y, E_{i}, F$ and $H$, respectively. In what follows, it is assumed that $J_{E_{i}}(X), J_{F}(X)$ and $J(H(Y) Y \mid Y)$ exist and are symmetric and continuous.

It should also be noted that this work was inspired essentially by the papers of Tunç $[14,15,16]$. We study the theoretical aspect of the subject and all the papers mentioned above have focused on the special cases of Eq. (1). This paper is also the first attempt to investigate the instability to vector functional differential equations of the fourth order with multiple deviating arguments, and it has a contribution to the subject in the literature and may be useful for researchers working on the qualitative behaviors of solutions. Based on Krasovskii's criterions (see [5]), we prove our main result.

The symbol $\langle X, Y\rangle$ corresponding to any pair $X, Y$ in $\Re^{n}$ stands for the usual scalar $\langle X, Y\rangle=\sum_{i=1}^{n} x_{i} y_{i}$; thus $\langle X, X\rangle=\|X\|^{2}$, and $\lambda_{i}(\Omega),(i=$ $1,2, \ldots, n)$, are the eigenvalues of the real symmetric $n \times n-$ matrix $\Omega$. The matrix $\Omega$ is said to be negative semi-definite, when $\langle\Omega X, X\rangle \leq 0$ for all nonzero $X$ in $\Re^{n}$.

\section{Main results}

Before introduction of the main result, we need the following results.

Lemma 1 (Bellman [1]). Let $A$ be a real symmetric $n \times n$-matrix and

$$
a^{\prime} \geq \lambda_{i}(A) \geq a>0, \quad(i=1,2, \ldots, n),
$$

where $a^{\prime}$ and $a$ are constants. Then

$$
a^{\prime}\langle X, X\rangle \geq\langle A X, X\rangle \geq a\langle X, X\rangle
$$

and

$$
{a^{\prime}}^{2}\langle X, X\rangle \geq\langle A X, A X\rangle \geq a^{2}\langle X, X\rangle
$$


Let $r \geq 0$ be given, and let $C=C\left([-r, 0], \Re^{n}\right)$ with

$$
\|\phi\|=\max _{-r \leq s \leq 0}|\phi(s)|, \quad \phi \in C .
$$

For $H>0$ define $C_{H} \subset C$ by

$$
C_{H}=\{\phi \in C:\|\phi\|<H\} .
$$

If $x:[-r, A) \rightarrow \Re^{n}$ is continuous, $0<A \leq \infty$, then, for each $t$ in $[0, A)$, $x_{t}$ in $C$ is defined by

$$
x_{t}(s)=x(t+s), \quad-r \leq s \leq 0, \quad t \geq 0 .
$$

Let $G$ be an open subset of $C$ and consider the general autonomous delay differential system with finite delay

$$
\dot{x}=F\left(x_{t}\right), \quad x_{t}=x(t+\theta), \quad-r \leq \theta \leq 0, t \geq 0,
$$

where $F: G \rightarrow \Re^{n}$ is continuous and maps closed and bounded sets into bounded sets. It follows from these conditions on $F$ that each initial value problem

$$
\dot{x}=F\left(x_{t}\right), \quad x_{0}=\phi \in G
$$

has a unique solution defined on some interval $[0, A), 0<A \leq \infty$. This solution will be denoted by $x(\phi)(\cdot)$ so that $x_{0}(\phi)=\phi$.

Definition 1. The zero solution, $x=0$, of $\dot{x}=F\left(x_{t}\right)$ is stable if for each $\varepsilon>0$ there exists $\delta=\delta(\varepsilon)>0$ such that $\|\phi\|<\delta$ implies that $|x(\phi)(t)|<\varepsilon$ for all $t \geq 0$. The zero solution is said to be unstable if it is not stable.

The main result of this paper is the following theorem.

Theorem 1. In addition to the basic assumptions imposed on $A, H$, $G, F$ and $E_{i}$ that appear in Eq. (1), we assume that there exist a negative constant $a_{1}$ and positive constants $a_{3}, a_{4}, \alpha_{i}, k, \beta_{i}$ and $\rho$ such that the following conditions hold:

$$
\begin{gathered}
\lambda_{i}(A) \leq a_{1}, F(0)=0, F(X) \neq 0,(X \neq 0), 0<\lambda_{i}\left(J_{F}(X)\right) \leq a_{4}, \\
E_{i}(0)=0, E_{i}(X) \neq 0,(X \neq 0), 0<\lambda_{i}\left(J_{E_{i}}(X)\right) \leq \beta_{i}
\end{gathered}
$$

and

$$
\lambda_{i}(G(X)) \geq a_{3} \text { for all } X \in \Re^{n} .
$$

If

$$
\tau^{*}<\frac{a_{3}}{k+2 \rho},
$$

then the solution $X=0$ of Eq. (1) is unstable for arbitrary $H(Y)$. 
Remark 1. It should be noted that there is no sign restriction on eigenvalues of the matrix $H(Y)$ in (2), and it is clear that our assumptions have a very simple form and the applicability of them can be easily followed.

Proof. Define the Lyapunov-Krasovskii functional $V(\cdot)=V\left(X_{t}, Y_{t}, Z_{t}\right.$, $\left.U_{t}\right)$

$$
\begin{aligned}
V(\cdot)= & -\langle A Y, Z\rangle-\langle Y, U\rangle+\frac{1}{2}\langle Z, Z\rangle \\
& -\int_{0}^{1} \sigma\langle H(\sigma Y) Y, Y\rangle d \sigma-\int_{0}^{1}\langle F(\sigma X), X\rangle d \sigma \\
& -\int_{0}^{1} \sum_{i=1}^{n}\left\langle E_{i}(\sigma X), X\right\rangle d \sigma-k \int_{-\tau}^{0} \int_{t+s}^{t}\|Y(\theta)\|^{2} d \theta d s \\
& -\sum_{i=1}^{n} k_{i} \int_{-\tau_{i}}^{0} \int_{t+s}^{t}\|Y(\theta)\|^{2} d \theta d s,
\end{aligned}
$$

where $k$ and $k_{i}$ are certain positive constants to be determined later.

It follows that $V(0,0,0,0)=0$.

Let

$$
\bar{\varepsilon}=\left(\varepsilon_{31}, \ldots, \varepsilon_{3 n}\right)
$$

so that

$$
V(0,0, \bar{\varepsilon}, 0)=\frac{1}{2}\langle\bar{\varepsilon}, \bar{\varepsilon}\rangle=\frac{1}{2}\|\bar{\varepsilon}\|^{2}>0
$$

for all arbitrary $\bar{\varepsilon} \neq 0, \bar{\varepsilon} \in \Re^{n}$. Thus, the functional $V(\cdot)$ satisfies the property $\left(K_{1}\right)$ of Krasovskii [5].

Let $(X, Y, Z, U)=(X(t), Y(t), Z(t), U(t))$ be an arbitrary solution of $(2)$. The time derivative of $V(\cdot)$ along $(2)$ gives

$$
\begin{aligned}
\frac{d}{d t} V(\cdot)= & -\langle A Z, Z\rangle+\langle G(X) Y, Y\rangle-<\int_{t-\tau}^{t} J_{F}(X(s)) Y(s) d s, Y> \\
& -<\sum_{i=1}^{n} \int_{t-\tau_{i}}^{t} J_{E_{i}}(X(s)) Y(s) d s, Y>-k \tau\|Y\|^{2} \\
& +k \int_{t-\tau}^{t}\|Y(\theta)\|^{2} d \theta-\sum_{i=1}^{n}\left(k_{i} \tau_{i}\right)\|Y\|^{2} \\
& +\sum_{i=1}^{n} k_{i} \int_{t-\tau_{i}}^{t}\|Y(\theta)\|^{2} d \theta .
\end{aligned}
$$

It can be easily seen that

$$
-<\int_{t-\tau}^{t} J_{F}(X(s)) Y(s) d s, Y>\geq-\|Y\|\left\|\int_{t-\tau}^{t} J_{F}(X(s)) Y(s) d s\right\|
$$




$$
\begin{aligned}
& \geq-\sqrt{n} a_{4}\|Y\|\left\|\int_{t-\tau}^{t} Y(s)\right\| d s \\
& \geq-\sqrt{n} a_{4}\|Y\| \int_{t-\tau}^{t}\|Y(s)\| d s \\
& \geq-\frac{1}{2} \sqrt{n} a_{4} \int_{t-\tau}^{t}\left(\|Y(t)\|^{2}+\|Y(s)\|^{2}\right) d s \\
& \geq-\frac{1}{2} \sqrt{n} a_{4} \tau\|Y\|^{2}-\frac{1}{2} \sqrt{n} a_{4} \int_{t-\tau}^{t}\|Y(s)\|^{2} d s
\end{aligned}
$$

and

$$
\begin{aligned}
<\int_{t-\tau_{i}}^{t} J_{E_{i}}(X(s)) Y(s) d s, Y> & \geq-\|Y\|\left\|\int_{t-\tau_{i}}^{t} J_{E_{i}}(X(s)) Y(s) d s\right\| \\
& \geq-\sqrt{n} \beta_{i}\|Y\|\left\|\int_{t-\tau_{i}}^{t} Y(s)\right\| d s \\
& \geq-\sqrt{n} \beta_{i}\|Y\| \int_{t-\tau_{i}}^{t}\|Y(s)\| d s \\
& \geq-\frac{1}{2} \sqrt{n} \beta_{i} \int_{t-\tau_{i}}^{t}\left(\|Y(t)\|^{2}+\|Y(s)\|^{2}\right) d s \\
& \geq-\frac{1}{2} \sqrt{n} \beta_{i} \tau_{i}\|Y\|^{2}-\frac{1}{2} \sqrt{n} \beta_{i} \int_{t-\tau_{i}}^{t}\|Y(s)\|^{2} d s
\end{aligned}
$$

so that

$$
\begin{aligned}
\frac{d}{d t} V(\cdot) \geq & -\langle A Z, Z\rangle+\langle G(X) Y, Y\rangle-\frac{1}{2} \sqrt{n} a_{4} \tau\|Y\|^{2} \\
& -\frac{1}{2} \sqrt{n} \sum_{i=1}^{n}\left(\beta_{i} \tau_{i}\right)\|Y\|^{2}-k \tau\|Y\|^{2}-\sum_{i=1}^{n}\left(k_{i} \tau_{i}\right)\|Y\|^{2} \\
& +\left(k-\frac{1}{2} \sqrt{n} a_{4}\right) \int_{t-\tau}^{t}\|Y(s)\|^{2} d s \\
& +\left(\sum_{i=1}^{n} k_{i}-\frac{1}{2} \sqrt{n} \sum_{i=1}^{n} \beta_{i}\right) \int_{t-\tau_{i}}^{t}\|Y(\theta)\|^{2} d \theta \\
\geq & -a_{1}\|Z\|^{2}+\left\{a_{3}-\left(\sum_{i=1}^{n}\left(k_{i} \tau_{i}\right)+k \tau+\frac{1}{2} \sqrt{n} \sum_{i=1}^{n} \beta_{i} \tau_{i}\right.\right. \\
& \left.\left.+\frac{1}{2} \sqrt{n} a_{4} \tau\right)\right\}\|Y\|^{2}+\left(k-\frac{1}{2} \sqrt{n} a_{4}\right) \int_{t-\tau}^{t}\|Y(s)\|^{2} d s \\
& +\left(\sum_{i=1}^{n} k_{i}-\frac{1}{2} \sqrt{n} \beta_{i}\right) \int_{t-\tau_{i}}^{t}\|Y(s)\|^{2} d s .
\end{aligned}
$$


Then, using the estimate $\tau^{*}=\max \left(\tau_{i}, \tau\right)$, we get

$$
\begin{aligned}
\frac{d}{d t} V(\cdot) \geq & -a_{1}\|Z\|^{2}+\left\{a_{3}-\left(\sum_{i=1}^{n} k_{i}+k+\frac{1}{2} \sqrt{n} \sum_{i=1}^{n}\left(\beta_{i}+a_{4}\right)\right) \tau^{*}\right\}\|Y\|^{2} \\
& +\left(k-\frac{1}{2} \sqrt{n} a_{4}\right) \int_{t-\tau}^{t}\|Y(s)\|^{2} d s \\
& +\left(\sum_{i=1}^{n} k_{i}-\frac{1}{2} \sqrt{n}\left(a_{4}+\beta_{i}\right) \int_{t-\tau}^{t}\|Y(s)\|^{2} d s .\right.
\end{aligned}
$$

Let

$$
\rho=\sum_{i=1}^{n} k_{i} \quad \text { and } \quad \sigma=\frac{1}{2} \sqrt{n}\left(\sum_{i=1}^{n} \beta_{i}+a_{4}\right) .
$$

Then, we have

$$
\begin{aligned}
\frac{d}{d t} V(\cdot) \geq & -a_{1}\|Z\|^{2}+\left\{a_{3}-(\rho+k+\sigma) \tau^{*}\right\}\|Y\|^{2} \\
& +\left(k-\frac{1}{2} \sqrt{n} a_{4}\right) \int_{t-\tau}^{t}\|Y(s)\|^{2} d s+(\rho-\sigma) \int_{t-\tau}^{t}\|Y(s)\|^{2} d s .
\end{aligned}
$$

Let

$$
k=\frac{1}{2} \sqrt{n} a_{4}, \quad \rho=\sigma .
$$

Hence

$$
\frac{d}{d t} V(\cdot) \geq-a_{1}\|Z\|^{2}+\left(a_{3}-(k+2 \rho) \tau^{*}\right)\|Y\|^{2} .
$$

If $\tau^{*}<\frac{a_{3}}{k+2 \rho}$, then we have for some positive constant $k^{*}$ that

$$
\frac{d}{d t} V(\cdot) \geq k^{*}\|Y\|^{2} \geq 0
$$

Then, it follows that the functional $V(\cdot)$ satisfies the property $\left(K_{2}\right)$ of Krasovskii [5].

On the other hand, it follows that

$$
\frac{d}{d t} V(\cdot)=0 \Leftrightarrow Y=\dot{X}, Z=\dot{Y}=0, U=\dot{Z}=0 \text { for all } t \geq 0 .
$$

Hence

$$
X=\xi, \quad Y=Z=U=0 .
$$

Replacing the above estimates in $(2)$, we get that $F(\xi)+E_{i}(\xi)=0$. By the assumptions of the theorem, it follows that

$$
F(\xi)+E_{i}(\xi)=0 \Leftrightarrow \xi=0 .
$$


Then, we conclude that

$$
X=Y=Z=U=0 \text { for all } t \geq 0
$$

Hence, the functional $V($.$) satisfies the property \left(K_{3}\right)$ of Krasovskii [5]. The proof of the theorem is completed.

Example 1. In a special case of Eq. (1), for $n=2$, we choose

$$
\begin{gathered}
A=\left[\begin{array}{cc}
-6 & 2 \\
2 & -6
\end{array}\right] \\
G(X)=\left[\begin{array}{cc}
5+\frac{1}{1+x_{1}^{2}} & 0 \\
0 & 5+\frac{1}{1+x_{2}^{2}}
\end{array}\right], \\
F(X)=\left[\begin{array}{l}
3 x_{1} \\
2 x_{2}
\end{array}\right], \\
E_{1}\left(X\left(t-\tau_{1}\right)\right)=\left[\begin{array}{l}
2 x_{1}\left(t-\tau_{1}\right) \\
x_{2}\left(t-\tau_{1}\right)
\end{array}\right]
\end{gathered}
$$

and

$$
E_{2}\left(X\left(t-\tau_{2}\right)\right)=\left[\begin{array}{c}
4 x_{1}\left(t-\tau_{2}\right) \\
3 x_{2}\left(t-\tau_{2}\right)
\end{array}\right] .
$$

Then, by an easy calculation, we obtain

$$
\begin{gathered}
\lambda_{1}(A)=-4, \quad \lambda_{2}(A)=-8, \\
\lambda_{1}(G)=5+\frac{1}{1+x_{1}^{2}}, \quad \lambda_{2}(G)=5+\frac{1}{1+x_{2}^{2}}, \\
J_{F}(X)=\left[\begin{array}{cc}
3 & 0 \\
0 & 2
\end{array}\right], \\
J_{E_{1}}(X)=\left[\begin{array}{ll}
2 & 0 \\
0 & 1
\end{array}\right]
\end{gathered}
$$

and

$$
J_{E_{2}}(X)=\left[\begin{array}{ll}
4 & 0 \\
0 & 3
\end{array}\right]
$$

so that

$$
\begin{gathered}
\lambda_{i}(A) \leq-4=a_{1}, \\
\lambda_{i}(G) \geq 5=a_{3}>0, \\
0<\lambda_{i}\left(J_{F}(X)\right) \leq 3=a_{4},
\end{gathered}
$$




$$
0<\lambda_{i}\left(J_{E_{1}}(X)\right) \leq 2=\beta_{1}
$$

and

$$
0<\lambda_{i}\left(J_{E_{2}}(X)\right) \leq 4=\beta_{2}, \quad(i=1,2)
$$

If

$$
\tau^{*}<\frac{a_{3}}{k+2 \rho}=\frac{a_{3}}{k+\sqrt{n}\left(\sum_{i=1}^{n} \beta_{i}+a_{4}\right)}=\frac{5}{21 \sqrt{2}},
$$

then all the conditions of the Theorem 1 hold.

\section{References}

[1] Bellman R., Introduction to matrix analysis, Reprint of the second (1970) edition. With a foreword by Gene Golub. Classics in Applied Mathematics, 19. Society for Industrial and Applied Mathematics (placecountry-regionSIAM), CityplacePhiladelphia, StatePA, 1997.

[2] EzeILO J.O.C., An instability theorem for a certain fourth order differential equation, Bull. London Math. Soc., 10(2)(1978), 184-185.

[3] EzEILO J.O.C., Extension of certain instability theorems for some fourth and fifth order differential equations, Atti. Accad. Naz. Lincei. Rend. Cl. Sci. Fis. Mat. Natur., (8)66(4)(1979), 239-242.

[4] EzEILO J.O.C., Further instability theorems for some fourth order differential equations, J. Nigerian Mathematical Society, 19(2000), 1-7.

[5] Krasovskit N.N., On conditions of inversion of A. M. Lyapunov's theorems on instability for stationary systems of differential equations (Russian), Dokl. Akad. Nauk. SSSR (N.S.), 101(1955), 17-20.

[6] SADEK A.I., Instability results for certain systems of fourth and fifth order differential equations, Appl. Math. Comput., 145(2-3)(2003), 541-549.

[7] Sun W.J., Hou X, New results about instability of some fourth and fifth order nonlinear systems (Chinese), J. Xinjiang Univ. Natur. Sci., 16(4)(1999), 14-17.

[8] TUNÇ C., An instability theorem for a certain vector differential equation of the fourth order, J. Inequal. Pure Appl. Math., 5(1)(2004), 1-5.

[9] TunÇ C., A further instability result for a certain vector differential equation of fourth order, Int. J. Math. Game Theory Algebra, 15(5)(2006), 489-495.

[10] TunÇ C., Instability of solutions for certain nonlinear vector differential equations of fourth order, Nonlinear Oscil. (N. Y.), 12(1)(2009), 120-129.

[11] Tunç C., On the instability of the solutions of some nonlinear vector differential equations of fourth order, Miskolc Math. Notes, 11(2)(2010), 191-200.

[12] TunÇ C., Recent advances on instability of solutions of fourth and fifth order delay differential equations with some open problems, World Scientific Review, Vol. 9, World Scientific Series on Nonlinear Science Series B (Book Series), (2011), 105-116.

[13] Tunç C., On the instability of solutions of nonlinear delay differential equations of fourth and fifth order, Sains Malaysiana, 40(12)(2011), 1455-1459.

[14] TunÇ C., On the instability of solutions of a nonlinear vector differential equation of fourth order, Ann. Differential Equations, 27(4)(2011), 418-421. 
[15] TunÇ C., Instability for nonlinear differential equations of fifth order subject to delay, Nonlinear Dyn. Syst. Theory, 12(2)(2012), 207-214.

[16] Tunç C., Instability to nonlinear vector differential equations of fourth order with constant delay, Sains Malaysiana, 42(7)(2013).

\author{
Cemil TunÇ \\ Department of Mathematics \\ FACUlTy OF SCIENCES \\ YÜZÜNCÜ YIL UNIVERSITY \\ 65080, VAN, TURKEY \\ e-mail: cemtunc@yahoo.com
}

Received on 04.11.2013 and, in revised form, on 19.11.2015. 